\title{
Quartic B-Spline Collocation Method for Eighth Order Boundary Value Problems
}

\author{
Y. Showri Raju \\ Associate Professor, Department of Computational Sciences, College of Natural and Computational Sciences \\ Wollega University, Nekemte, Ethiopia \\ *Corresponding Author: Y. Showri Raju, Associate Professor, Department of Computational Sciences, \\ College of Natural and Computational Sciences Wollega University, Nekemte, Ethiopia
}

\begin{abstract}
A finite element method involving collocation method with Quartic B-splines as basis functions has been developed to solve eighth order boundary value problems. The fourth order, fifth order, sixth order, seventh order and eighth order derivatives for the dependent variable are approximated by the finite differences of third order derivatives. The basis functions are redefined into a new set of basis functions which in number match with the number of collocated points selected in the space variable domain. The proposed method is tested on three linear and two non-linear boundary value problems. The solution of a non-linear boundary value problem has been obtained as the limit of a sequence of solutions of linear boundary value problems generated by quasi linearization technique. Numerical results obtained by the present method are in good agreement with the exact solutions available in the literature.
\end{abstract}

Keywords: collocation method; quartic B-spline; basis function; eighth order boundary value problem; absolute error

\section{INTRODUCTION}

Generally, the eighth-order boundary value problems are known to arise in the Mathematics, Physics and Engineering Sciences [1, 2]. In the book written by Chandrasekhar [3], we can find that when an infinite horizontal layer of fluid is heated from below and is under the action of rotation, instability sets in. When this instability sets as an ordinary convection, the ordinary differential equation is a sixth-order ordinary differential equation. When this instability sets as an over stability, it is modelled by an eighth-order ordinary differential equation. An eighth-order differential equation occurring in torsional vibration of uniform beams was investigated by [4]. In this paper, we developed a collocation method with quartic B-splines as basis functions for getting the numerical solution of a general linear eighth order boundary value problem

$$
\begin{aligned}
& a_{0}(x) y^{(8)}(x)+a_{1}(x) y^{(7)}(x)+a_{2}(x) y^{(6)}(x)+a_{3}(x) y^{(5)}(x)+a_{4}(x) y^{(4)}(x)+a_{5}(x) y^{\prime \prime \prime}(x) \\
& +a_{6}(x) y^{\prime \prime}(x)+a_{7}(x) y^{\prime}(x)+a_{8}(x) y(x)=b(x), \quad c<x<d
\end{aligned}
$$

subject to the boundary conditions

$$
\begin{array}{ll}
y(c)=A_{0}, & y(d)=B_{0}, \\
y^{\prime}(c)=A_{1}, & y^{\prime}(d)=B_{1}, \\
y^{\prime \prime}(c)=A_{2}, & y^{\prime \prime}(d)=B_{2}, \\
y^{\prime \prime \prime}(c)=A_{3}, & y^{\prime \prime \prime}(d)=B_{3},
\end{array}
$$

where $A_{0}, B_{0}, A_{1}, B_{1}, A_{2}, B_{2}, A_{3}, B_{3}$ are finite real constants and $a_{0}(x), a_{1}(x), a_{2}(x), a_{3}(x), a_{4}(x), a_{5}(x)$, $a_{6}(x), a_{7}(x), a_{8}(x)$ and $b(x)$ are all continuous functions defined on the interval [c,d]. 
The existence and uniqueness of solution of such type of boundary value problems can be found in the book written by Agarwal [5]. Over the years, there are several authors who worked on these types of boundary value problems by using different methods. For example, Boutayeb and Twizell [6] developed finite difference methods for the solution of eighth-order boundary value problems. Twizell et al. [7] developed numerical methods for eighth, tenth and twelfth order eigenvalue problems arising in thermal instability. Siddiqi and Twizell [8] presented the solution of eighth order boundary value problem using octic spline. Inc and Evans [9] presented the solutions of eighth order boundary value problems using adomian decomposition method. Siddiqi and Ghazala Akram [10, 11] presented the solutions of eighth-order linear special case boundary value problems using nonic spline and nonpolynomial nonic spline respectively.

Further, Scott and Watts [12] developed a numerical method for the solution of linear boundary value problems using a combination of superposition and orthonormalization. Scott and Watts [13] described several computer codes that were developed using the superposition and orthonormalization technique and invariant imbedding. Watson and Scott [14] proved that Chow-Yorke algorithm was globally convergent for a class of spline collocation approximations to nonlinear two point boundary value problems. Liu and $\mathrm{Wu}$ [15] presented differential quadrature solutions of eighth-order differential equations. He $[16,17,18,19,20]$ developed the variational iteration technique for solving non linear initial and boundary value problems. Wazwaz [21] have used the modified decomposition method for approximating solution of higher-order boundary value problems with two point boundary conditions. Modified adomian decomposition method was used in [22] to find the analytical solution of linear and nonlinear boundary value problems of eighth order.

The above studies are concerned to solve eighth order boundary value problems by using octic or nonic B-splines. In this paper, quartic B-splines as basis functions have been used to solve the boundary value problems of the type (1)-(2).

In section 2 of this paper, the justification for using the collocation method has been mentioned. In section 3, the definition of quartic B-splines has been described. In section 4, description of the collocation method with quartic B-splines as basis functions has been presented and in section 5, solution procedure to find the nodal parameters is presented. In section 6, numerical examples of both linear and non-linear boundary value problems are presented. The solution of a nonlinear boundary value problem has been obtained as the limit of a sequence of solutions of linear boundary value problems generated by quasi linearization technique [23]. Finally, the last section is dealt with conclusions of the paper.

\section{JUSTIFICATION FOR USING COLlOCATION METHOD}

In finite element method (FEM) the approximate solution can be written as a linear combination of basis functions which constitute a basis for the approximation space under consideration. FEM involves variational methods such as Ritz's approach, Galerkin's approach, least squares method and collocation method etc. The collocation method seeks an approximate solution by requiring the residual of the differential equation to be identically zero at $\mathrm{N}$ selected points in the given space variable domain where $\mathrm{N}$ is the number of basis functions in the basis [24]. That means, to get an accurate solution by the collocation method, one needs a set of basis functions which in number match with the number of collocation points selected in the given space variable domain. Further, the collocation method is the easiest to implement among the variational methods of FEM. When a differential equation is approximated by $m^{\text {th }}$ order B-splines, it yields $(m+1)^{\text {th }}$ order accurate results [25]. Hence this motivated us to use the collocation method to solve a eighth order boundary value problem of type (1)-(2) with quartic B-splines.

\section{DEFINITION OF QUARTIC B-SPLINES}

The cubic B-splines are defined in [26, 27]. In a similar analogue, the existence of the quartic spline interpolate $\mathrm{s}(\mathrm{x})$ to a function in a closed interval $[\mathrm{c}, \mathrm{d}]$ for spaced knots (need not be evenly spaced)

$$
\mathrm{c}=\mathrm{x}_{0}<\mathrm{x}_{1}<\mathrm{x}_{2}<\ldots<\mathrm{x}_{\mathrm{n}-1}<\mathrm{x}_{\mathrm{n}}=\mathrm{d}
$$

is established by constructing it. The construction of $\mathrm{s}(\mathrm{x})$ is done with the help of the quartic BSplines. Introduce eight additional knots $\mathrm{x}_{-4}, \mathrm{x}_{-3}, \mathrm{x}_{-2}, \mathrm{x}_{-1}, \mathrm{x}_{\mathrm{n}+1}, \mathrm{x}_{\mathrm{n}+2}, \mathrm{x}_{\mathrm{n}+3}$ and $\mathrm{x}_{\mathrm{n}+4}$ such that 
$\mathrm{x}_{-4}<\mathrm{x}_{-3}<\mathrm{x}_{-2}<\mathrm{x}_{-1}<\mathrm{x}_{0}$ and $\mathrm{x}_{\mathrm{n}}<\mathrm{x}_{\mathrm{n}+1}<\mathrm{x}_{\mathrm{n}+2}<\mathrm{x}_{\mathrm{n}+3}<\mathrm{x}_{\mathrm{n}+4}$.

Now the quartic $\mathrm{B}$-splines $\mathrm{B}_{\mathrm{i}}(\mathrm{x})$ are defined by

$$
\begin{aligned}
& B_{i}(x)= \begin{cases}\sum_{r=i-2}^{i+3} \frac{\left(x_{r}-x\right)^{4}+}{\pi^{\prime}\left(x_{r}\right)} & x \in\left[x_{i-2}, x_{i+3}\right] \\
0 & \text { otherwise }\end{cases} \\
& \text { where } \\
& \left(x_{r}-x\right)^{4}+\left\{\begin{array}{lll}
\left(x_{r}-x\right)^{4}, & \text { if } & x_{r} \geq x \\
0, & \text { if } & x_{r} \leq x
\end{array}\right. \\
& \text { and } \pi(x)=\prod_{r=i-2}^{i+3}\left(x-x_{r}\right) .
\end{aligned}
$$

It can be shown that the set $\left\{\mathrm{B}_{-2}(\mathrm{x}), \mathrm{B}_{-1}(\mathrm{x}), \mathrm{B}_{0}(\mathrm{x}), \ldots, \mathrm{B}_{\mathrm{n}}(\mathrm{x}), \mathrm{B}_{\mathrm{n}+1}(\mathrm{x})\right\}$ forms a basis for the space $S_{4}(\pi)$ of quartic polynomial splines. The quartic B-splines are the unique non-zero splines of smallest compact support with knots at

$\mathrm{x}_{-4}<\mathrm{x}_{-3}<\mathrm{x}_{-2}<\mathrm{x}_{-1}<\mathrm{x}_{0}<\ldots<\mathrm{x}_{\mathrm{n}}<\mathrm{x}_{\mathrm{n}+1}<\mathrm{x}_{\mathrm{n}+2}<\mathrm{x}_{\mathrm{n}+3}<\mathrm{x}_{\mathrm{n}+4}$

\section{DESCRIPTION OF THE METHOD}

To solve the boundary value problem (1)-(2) by the collocation method with quartic B-splines as basis functions, we define the approximation for $y(x)$ as

$$
y(x)=\sum_{j=-2}^{n+1} \alpha_{j} B_{j}(x)
$$

where $\alpha_{j} s$ are the nodal parameters to be determined. In the present method, the internal mesh points $x_{2}, x_{3}, \ldots, x_{n-3}$ are selected as the collocation points. In collocation method, the number of basis functions in the approximation should match with the number of selected collocation points [24]. Here the number of basis functions in the approximation (3) is $n+4$, where as the number of selected collocation points is $\mathrm{n}-4$. So, there is a need to redefine the basis functions into a new set of basis functions which in number match with the number of selected collocation points. The procedure for redefining the basis functions is as follows:

Using the quartic B-splines described in section 3 and the Dirichlet boundary conditions of (2), we get the approximate solution at the boundary points as

$$
\begin{gathered}
y(c)=y\left(x_{0}\right)=\sum_{j=-2}^{1} \alpha_{j} B_{j}\left(x_{0}\right)=A_{0} \\
y(d)=y\left(x_{n}\right)=\sum_{j=n-2}^{n+1} \alpha_{j} B_{j}\left(x_{n}\right)=B_{0} .
\end{gathered}
$$

Eliminating $\alpha_{-2}$ and $\alpha_{n+1}$ from the equations (3), (4) and (5), we get the approximation for

$$
y(x) \text { as } \quad y(x)=w_{1}(x)+\sum_{j=-1}^{n} \alpha_{j} P_{j}(x)
$$

where

$$
w_{1}(x)=\frac{A_{0}}{B_{-2}\left(x_{0}\right)} B_{-2}(x)+\frac{B_{0}}{B_{n+1}\left(x_{n}\right)} B_{n+1}(x)
$$

and

$$
P_{j}(x)=\left\{\begin{array}{cc}
B_{j}(x)-\frac{B_{j}\left(x_{0}\right)}{B_{-2}\left(x_{0}\right)} B_{-2}(x), & \text { for } i=-1,0,1 \\
B_{j}(x), & \text { for } j=2,3, \ldots, n-3 \\
B_{j}(x)-\frac{B_{j}\left(x_{n}\right)}{B_{n+1}\left(x_{n}\right)} B_{n+1}(x), & \text { for } j=n-2, n-1, n .
\end{array}\right.
$$


Using the Neumann boundary conditions of (2) to the approximate solution $y(x)$ in (6), we get

$$
\begin{aligned}
& y^{\prime}(c)=y^{\prime}\left(x_{0}\right)=w_{1}^{\prime}\left(x_{0}\right)+\alpha_{-1} P_{-1}^{\prime}\left(x_{0}\right)+\alpha_{0} P_{0}^{\prime}\left(x_{0}\right)+\alpha_{1} P_{1}^{\prime}\left(x_{0}\right)=A_{1} \\
& y^{\prime}(d)=y^{\prime}\left(x_{n}\right)=w_{1}^{\prime}\left(x_{n}\right)+\alpha_{n-2} P_{n-2}^{\prime}\left(x_{n}\right)+\alpha_{n-1} P_{n-1}^{\prime}\left(x_{n}\right)+\alpha_{n} P_{n}^{\prime}\left(x_{n}\right)=B_{1} .
\end{aligned}
$$

Now, eliminating $\alpha_{-1}$ and $\alpha_{n}$ from the equations (6), (7) and(8), we get the approximation for $y(x)$ as

$y(x)=w_{2}(x)+\sum_{j=0}^{n-1} \alpha_{j} Q_{j}(x)$

Where

$w_{2}(x)=w_{1}(x)+\frac{A_{1}-w_{1}^{*}\left(x_{0}\right)}{P_{-1}^{\prime}\left(x_{0}\right)} P_{-1}(x)+\frac{B_{1}-w_{1}^{*}\left(x_{n}\right)}{P_{n}^{v}\left(x_{n}\right)} P_{n}(x)$

And

$$
Q_{j}(x)=\left\{\begin{array}{cc}
P_{j}(x)-\frac{P_{j}^{\prime}\left(x_{0}\right)}{P_{-1}^{j}\left(x_{0}\right)} P_{-1}(x), & \text { for } \quad \mathrm{j}=0,1 \\
P_{j}(x), & \text { for } \mathrm{j}=2,3, \ldots, n-3 \\
P_{j}(x)-\frac{P_{j}^{s}\left(x_{n}\right)}{P_{n}^{j}\left(x_{n}\right)} P_{n}(x), & \text { for } \mathrm{j}=n-2, n-1 .
\end{array}\right.
$$

Using the boundary conditions $y^{\prime \prime}(c)=A_{2}$ and $y^{\prime \prime}(d)=B_{2}$ of (2) to the approximate solution $y(x)$ in (9), we get

$y^{\prime \prime}(c)=y^{\prime \prime}\left(x_{0}\right)=w_{2}^{\prime \prime}\left(x_{0}\right)+\alpha_{0} Q_{0}^{\prime \prime}\left(x_{0}\right)+\alpha_{1} Q_{1}^{\prime \prime}\left(x_{0}\right)=A_{2}$

$y^{\prime \prime}(d)=y^{\prime \prime}\left(x_{n}\right)=w_{2}^{\prime \prime}\left(x_{n}\right)+\alpha_{n-2} Q_{n-2}^{\prime \prime}\left(x_{n}\right)+\alpha_{n-1} Q_{n-1}^{\prime \prime}\left(x_{n}\right)=B_{2}$.

Now, eliminating $\alpha_{0}$ and $\alpha_{n-1}$ from the equations (9), (10), and (11), we get the approximation for $y(x)$ as

$\mathrm{y}(\mathrm{x})=\mathrm{w}_{3}(\mathrm{x})+\sum_{j=1}^{n-2} \alpha_{j} \mathrm{R}_{\mathrm{j}}(\mathrm{x})$

where $\quad \mathrm{w}_{3}(\mathrm{x})=\mathrm{w}_{2}(\mathrm{x})+\frac{\mathrm{A}_{2}-\mathrm{w}_{2}{ }^{\prime \prime}\left(\mathrm{x}_{0}\right)}{\mathrm{Q}_{0}{ }^{\prime}\left(\mathrm{x}_{0}\right)} \mathrm{Q}_{0}(\mathrm{x})+\frac{\mathrm{B}_{2}-\mathrm{w}_{2}{ }^{\prime \prime}\left(\mathrm{x}_{\mathrm{n}}\right)}{\mathrm{Q}_{\mathrm{n}-1}\left(\mathrm{x}_{\mathrm{n}}\right)} \mathrm{Q}_{\mathrm{n}-1}(\mathrm{x})$

and

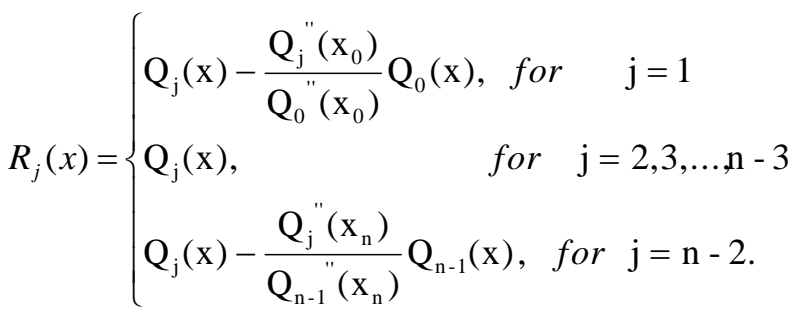

Now, using the boundary conditions $\mathrm{y}^{\prime \prime \prime}(\mathrm{c})=\mathrm{A}_{3}$ and $\mathrm{y}^{\prime \prime \prime}(\mathrm{d})=\mathrm{B}_{3}$ of (2) to the approximate solution $y(x)$ in (12), we get

$\mathrm{y}^{\prime \prime \prime}(\mathrm{c})=\mathrm{y}^{\prime \prime \prime}\left(\mathrm{x}_{0}\right)=\mathrm{w}_{3}{ }^{\prime \prime \prime}\left(\mathrm{x}_{0}\right)+\alpha_{1} \mathrm{R}_{1}{ }^{\prime \prime \prime}\left(\mathrm{x}_{0}\right)=\mathrm{A}_{3}$

$\mathrm{y}^{\prime \prime \prime}(\mathrm{d})=\mathrm{y}^{\prime \prime \prime}\left(\mathrm{x}_{\mathrm{n}}\right)=\mathrm{w}_{3}{ }^{\prime \prime \prime}\left(\mathrm{x}_{\mathrm{n}}\right)+\alpha_{\mathrm{n}-2} \mathrm{R}_{\mathrm{n}-2}{ }^{\prime \prime \prime}\left(\mathrm{x}_{\mathrm{n}}\right)=\mathrm{B}_{3}$.

Now, eliminating $\alpha_{1}$ and $\alpha_{\mathrm{n}-2}$ from the equations (12), (13) and (14), we get the approximation for $\mathrm{y}(\mathrm{x})$ as

$$
\mathrm{y}(\mathrm{x})=\mathrm{w}(\mathrm{x})+\sum_{\mathrm{j}=2}^{n-3} \alpha_{\mathrm{j}} \widetilde{\mathrm{B}}_{\mathrm{j}}(\mathrm{x})
$$


where

$$
\mathrm{w}(\mathrm{x})=\mathrm{w}_{3}(\mathrm{x})+\frac{\mathrm{A}_{3}-\mathrm{w}_{3}{ }^{\prime \prime}\left(\mathrm{x}_{0}\right)}{\mathrm{R}_{1}{ }^{\prime \prime}\left(\mathrm{x}_{0}\right)} \mathrm{R}_{1}(\mathrm{x})+\frac{\mathrm{B}_{3}-\mathrm{w}_{3}{ }^{\prime \prime}\left(\mathrm{x}_{\mathrm{n}}\right)}{\mathrm{R}_{\mathrm{n}-2}{ }^{\prime \prime}\left(\mathrm{x}_{\mathrm{n}}\right)} \mathrm{R}_{\mathrm{n}-2}(\mathrm{x})
$$

and

$$
\widetilde{\mathrm{B}}_{\mathrm{j}}(\mathrm{x})=R_{j}(x), \quad \text { for } \mathrm{j}=2,3, \ldots, \mathrm{n}-3 .
$$

Now the new basis functions for the approximation $y(x) \operatorname{are}\left\{\widetilde{B_{j}}(x), j=2,3, \ldots, n-3\right\}$ and they are in number matching with the number of selected collocated points. Since the approximation for $y(x)$ in (15) is a quartic approximation, let us approximate $y^{(4)}, y^{(5)}, y^{(6)}, y^{(7)}$ and $y^{(8)}$ at the selected collocation points with finite differences as

$y_{i}^{(8)}=\frac{y_{(i+5)}^{\prime \prime \prime}-5 y_{(i+4)}^{\prime \prime \prime}+10 y_{i+3}^{\prime \prime \prime}-10 y_{(i+2)}^{\prime \prime \prime}+5 y_{i+1}^{\prime \prime \prime}-y_{(i)}^{\prime \prime \prime}}{h^{5}}$ for $\mathrm{i}=2$

$y_{i}^{(8)}=\frac{y_{(i+3)}^{\prime \prime \prime}-4 y_{(i+2)}^{\prime \prime \prime}+5 y_{i+1}^{\prime \prime \prime}-5 y_{(i-1)}^{\prime \prime \prime}+4 y_{i-2}^{\prime \prime \prime}-y_{(i-3)}^{\prime \prime \prime}}{2 h^{5}}$ for $\mathrm{i}=3,4, \ldots, \mathrm{n}-3$

$y_{i}^{(7)}=\frac{y_{(i+2)}^{\prime \prime \prime}-4 y_{i+1}^{\prime \prime \prime}+6 y_{(i)}^{\prime \prime \prime}-4 y_{i-1}^{\prime \prime \prime}+y_{(i-2)}^{\prime \prime \prime}}{h^{4}}$ for $\mathrm{i}=2,3, \ldots, \mathrm{n}-3$

$y_{i}^{(6)}=\frac{y_{(i+2)}^{\prime \prime \prime}-2 y_{i+1}^{\prime \prime \prime}+2 y_{i-1}^{\prime \prime \prime}-y_{(i-2)}^{\prime \prime \prime}}{2 h^{3}}$ for $\mathrm{i}=2,3, \ldots, \mathrm{n}-3$

$y_{i}^{(5)}=\frac{y_{i+1}^{\prime \prime \prime}-2 y_{i}^{\prime \prime \prime}-y_{i-1}^{\prime \prime \prime}}{h^{2}}$ for $\mathrm{i}=2,3, \ldots, \mathrm{n}-3$

$y_{i}^{(4)}=\frac{y_{i+1}^{\prime \prime \prime}-y_{i-1}^{\prime \prime \prime}}{2 h}$ for $\mathrm{i}=2,3, \ldots, \mathrm{n}-3$

Where

$$
\mathrm{y}_{\mathrm{i}}=\mathrm{y}\left(\mathrm{x}_{\mathrm{i}}\right)=\mathrm{w}\left(\mathrm{x}_{\mathrm{i}}\right)+\sum_{\mathrm{j}=2}^{\mathrm{n}-3} \alpha_{\mathrm{j}} \widetilde{\mathrm{B}}_{\mathrm{j}}\left(\mathrm{x}_{\mathrm{i}}\right) \text {. }
$$

Now applying collocation method to (1), we get

$$
\begin{aligned}
& a_{0}\left(x_{i}\right) y_{i}^{(8)}+a_{1}\left(x_{i}\right) y_{i}^{(7)}+a_{2}\left(x_{i}\right) y_{i}^{(6)}+a_{3}\left(x_{i}\right) y_{i}^{(5)}+a_{4}\left(x_{i}\right) y_{i}^{(4)}+a_{5}\left(x_{i}\right) y_{i}^{\prime \prime \prime} \\
& +a_{6}\left(x_{i}\right) y_{i}^{\prime \prime}+a_{7}\left(x_{i}\right) y_{i}^{\prime}+a_{8}\left(x_{i}\right) y_{i}=b\left(x_{i}\right) \quad \text { for } \mathrm{i}=2,3, \ldots, \mathrm{n}-3 .
\end{aligned}
$$

Using (16), (18) to (21) in (23), we get

$$
\begin{aligned}
& \frac{a_{0}\left(x_{i}\right)}{h^{5}}\left[\begin{array}{c}
\left\{w^{\prime \prime \prime}\left(\mathrm{x}_{\mathrm{i}+5}\right)+\sum_{\mathrm{j}=2}^{\mathrm{n}-3} \alpha_{\mathrm{j}} \widetilde{\mathrm{B}}_{\mathrm{j}}^{\prime \prime \prime}\left(\mathrm{x}_{\mathrm{i}+5}\right)\right\}-5\left\{\mathrm{w}^{\prime \prime \prime}\left(\mathrm{x}_{\mathrm{i}+4}\right)+\sum_{\mathrm{j}=2}^{\mathrm{n}-3} \alpha_{\mathrm{j}} \widetilde{\mathrm{B}}_{\mathrm{j}}^{\prime \prime \prime}\left(\mathrm{x}_{\mathrm{i}+4}\right)\right\} \\
+10\left\{\mathrm{w}^{\prime \prime \prime}\left(\mathrm{x}_{\mathrm{i}+3}\right)+\sum_{\mathrm{j}=2}^{\mathrm{n}-3} \alpha_{\mathrm{j}} \tilde{\mathrm{B}}_{\mathrm{j}}^{\prime \prime \prime}\left(\mathrm{x}_{\mathrm{i}+3}\right)\right\}-10\left\{\mathrm{w}^{\prime \prime \prime}\left(\mathrm{x}_{\mathrm{i}+2}\right)+\sum_{\mathrm{j}=2}^{\mathrm{n}-3} \alpha_{\mathrm{j}} \tilde{\mathrm{B}}_{\mathrm{j}}^{\prime \prime \prime}\left(\mathrm{x}_{\mathrm{i}+2}\right)\right\} \\
+5\left\{\mathrm{w}^{\prime \prime \prime}\left(\mathrm{x}_{\mathrm{i}+1}\right)+\sum_{\mathrm{j}=2}^{\mathrm{n}-3} \alpha_{\mathrm{j}} \widetilde{\mathrm{B}}_{\mathrm{j}}^{\prime \prime \prime}\left(\mathrm{x}_{\mathrm{i}+1}\right)\right\}-\left\{\mathrm{w}^{\prime \prime \prime}\left(\mathrm{x}_{\mathrm{i}}\right)+\sum_{\mathrm{j}=2}^{\mathrm{n}-3} \alpha_{\mathrm{j}} \widetilde{\mathrm{B}}_{\mathrm{j}}^{\prime \prime \prime}\left(\mathrm{x}_{\mathrm{i}}\right)\right\}
\end{array}\right] \\
& +\frac{a_{1}\left(x_{i}\right)}{h^{4}}\left[\begin{array}{c}
\left\{w^{\prime \prime \prime}\left(\mathrm{x}_{\mathrm{i}+2}\right)+\sum_{\mathrm{j}=2}^{\mathrm{n}-3} \alpha_{\mathrm{j}} \widetilde{\mathrm{B}}_{\mathrm{j}}^{\prime \prime \prime}\left(\mathrm{x}_{\mathrm{i}+2}\right)\right\}-4\left\{\mathrm{w}^{\prime \prime \prime}\left(\mathrm{x}_{\mathrm{i}+1}\right)+\sum_{\mathrm{j}=2}^{\mathrm{n}-3} \alpha_{\mathrm{j}} \tilde{\mathrm{B}}_{\mathrm{j}}^{\prime \prime \prime}\left(\mathrm{x}_{\mathrm{i}+1}\right)\right\} \\
+6\left\{\mathrm{w}^{\prime \prime \prime}\left(\mathrm{x}_{\mathrm{i}}\right)+\sum_{\mathrm{j}=2}^{\mathrm{n}-3} \alpha_{\mathrm{j}} \tilde{\mathrm{B}}_{\mathrm{j}}^{\prime \prime \prime}\left(\mathrm{x}_{\mathrm{i}}\right)\right\}-4\left\{\mathrm{w}^{\prime \prime \prime}\left(\mathrm{x}_{\mathrm{i}-1}\right)+\sum_{\mathrm{j}=2}^{\mathrm{n}-3} \alpha_{\mathrm{j}} \tilde{\mathrm{B}}_{\mathrm{j}}^{\prime \prime \prime}\left(\mathrm{x}_{\mathrm{i}-1}\right)\right\} \\
+\left\{\mathrm{w}^{\prime \prime \prime}\left(\mathrm{x}_{\mathrm{i}-2}\right)+\sum_{\mathrm{j}=2}^{\mathrm{n}-3} \alpha_{\mathrm{j}} \tilde{\mathrm{B}}_{\mathrm{j}}^{\prime \prime \prime}\left(\mathrm{x}_{\mathrm{i}-2}\right)\right\}
\end{array}\right] \\
& +\frac{a_{2}\left(x_{i}\right)}{2 h^{3}}\left[\begin{array}{c}
\left\{w^{\prime \prime \prime}\left(x_{i+2}\right)+\sum_{j=2}^{n-3} a_{j} \widetilde{\mathrm{B}}_{j}^{\prime \prime \prime}\left(x_{i+2}\right)\right\}-2\left\{w^{\prime \prime \prime}\left(x_{i+1}\right)+\sum_{j=2}^{n-3} a_{j} \widetilde{B}_{j}^{\prime \prime \prime}\left(x_{i+1}\right)\right\} \\
+2\left\{w^{\prime \prime \prime}\left(x_{i-1}\right)+\sum_{j=2}^{n-3} a_{j} \widetilde{B}_{j}^{\prime \prime \prime}\left(x_{i-1}\right)\right\}-\left\{w^{\prime \prime \prime}\left(x_{i-2}\right)+\sum_{j=2}^{n-3} a_{j} \widetilde{B}_{j}^{\prime \prime \prime}\left(x_{i-2}\right)\right\}
\end{array}\right] \\
& +\frac{a_{3}\left(x_{i}\right)}{h^{2}}\left[\begin{array}{c}
\left\{w^{\prime \prime \prime}\left(x_{i+1}\right)+\sum_{j=2}^{n-3} \alpha_{j} \widetilde{B}_{j}^{\prime \prime \prime}\left(x_{i+1}\right)\right\}-2\left\{w^{\prime \prime \prime}\left(x_{i}\right)+\sum_{j=2}^{n-3} \alpha_{j} \widetilde{B}_{j}^{\prime \prime \prime}\left(x_{i}\right)\right\} \\
+\left\{w^{\prime \prime \prime}\left(x_{i-1}\right)+\sum_{j=2}^{n-3} \alpha_{j} \widetilde{B}_{j}^{\prime \prime \prime}\left(x_{i-1}\right)\right\}
\end{array}\right] \\
& +\frac{a_{4}\left(x_{i}\right)}{2 h}\left[\left\{w^{\prime \prime \prime}\left(\mathrm{x}_{\mathrm{i}+1}\right)+\sum_{\mathrm{j}=2}^{\mathrm{n}-3} \alpha_{\mathrm{j}} \tilde{\mathrm{B}}_{\mathrm{j}}^{\prime \prime \prime}\left(\mathrm{x}_{\mathrm{i}+1}\right)\right\}-\left\{\mathrm{w}^{\prime \prime \prime}\left(\mathrm{x}_{\mathrm{i}-1}\right)+\sum_{\mathrm{j}=2}^{\mathrm{n}-3} \alpha_{\mathrm{j}} \tilde{\mathrm{B}}_{\mathrm{j}}^{\prime \prime \prime}\left(\mathrm{x}_{\mathrm{i}-1}\right)\right\}\right] \\
& +\mathrm{a}_{5}\left(\mathrm{x}_{\mathrm{i}}\right)\left[\mathrm{w}^{\prime \prime \prime}\left(\mathrm{x}_{\mathrm{i}}\right)+\sum_{\mathrm{j}=2}^{\mathrm{n}-3} \alpha_{\mathrm{j}} \widetilde{\mathrm{B}}_{\mathrm{j}}^{\prime \prime \prime}\left(\mathrm{x}_{\mathrm{i}}\right)\right]+a_{6}\left(x_{i}\right)\left[\mathrm{w}^{\prime \prime}\left(\mathrm{x}_{\mathrm{i}}\right)+\sum_{\mathrm{j}=2}^{\mathrm{n}-3} \alpha_{\mathrm{j}} \tilde{\mathrm{B}}_{\mathrm{j}}^{\prime \prime}\left(\mathrm{x}_{\mathrm{i}}\right)\right]
\end{aligned}
$$




$$
\begin{aligned}
& +a_{7}\left(x_{i}\right)\left[\mathrm{w}^{\prime}\left(\mathrm{x}_{\mathrm{i}}\right)+\sum_{\mathrm{j}=2}^{\mathrm{n}-3} \alpha_{\mathrm{j}} \widetilde{\mathrm{B}}_{\mathrm{j}}^{\prime}\left(\mathrm{x}_{\mathrm{i}}\right)\right]+a_{8}\left(x_{i}\right)\left[w\left(x_{i}\right)+\sum_{j=2}^{n-2} \alpha_{j} \widetilde{B}_{j}\left(x_{i}\right)\right] \\
& =b\left(x_{i}\right) \quad \text { for } \mathrm{i}=2 .
\end{aligned}
$$

Now using (17) to (21) and (22) in (23), we get

$$
\begin{aligned}
& \frac{a_{0}\left(x_{i}\right)}{2 h^{5}}\left[\begin{array}{c}
\left\{\mathrm{w}^{\prime \prime \prime}\left(\mathrm{x}_{\mathrm{i}+5}\right)+\sum_{\mathrm{j}=2}^{\mathrm{n}-3} \alpha_{\mathrm{j}} \tilde{\mathrm{B}}_{\mathrm{j}}^{\prime \prime \prime}\left(\mathrm{x}_{\mathrm{i}+5}\right)\right\}-5\left\{\mathrm{w}^{\prime \prime \prime}\left(\mathrm{x}_{\mathrm{i}+4}\right)+\sum_{\mathrm{j}=2}^{\mathrm{n}-3} \alpha_{\mathrm{j}} \tilde{\mathrm{B}}_{\mathrm{j}}^{\prime \prime \prime}\left(\mathrm{x}_{\mathrm{i}+4}\right)\right\} \\
+10\left\{\mathrm{w}^{\prime \prime \prime}\left(\mathrm{x}_{\mathrm{i}+3}\right)+\sum_{\mathrm{j}=2}^{\mathrm{n}-3} \alpha_{\mathrm{j}} \tilde{\mathrm{B}}_{\mathrm{j}}^{\prime \prime \prime}\left(\mathrm{x}_{\mathrm{i}+3}\right)\right\}-10\left\{\mathrm{w}^{\prime \prime \prime}\left(\mathrm{x}_{\mathrm{i}+2}\right)+\sum_{\mathrm{j}=2}^{\mathrm{n}-3} \alpha_{\mathrm{j}} \tilde{\mathrm{B}}_{\mathrm{j}}^{\prime \prime \prime}\left(\mathrm{x}_{\mathrm{i}+2}\right)\right\} \\
+5\left\{\mathrm{w}^{\prime \prime \prime}\left(\mathrm{x}_{\mathrm{i}+1}\right)+\sum_{\mathrm{j}=2}^{\mathrm{n}-3} \alpha_{\mathrm{j}} \widetilde{\mathrm{B}}_{\mathrm{j}}^{\prime \prime \prime}\left(\mathrm{x}_{\mathrm{i}+1}\right)\right\}-\left\{\mathrm{w}^{\prime \prime \prime}\left(\mathrm{x}_{\mathrm{i}}\right)+\sum_{\mathrm{j}=2}^{\mathrm{n}-3} \alpha_{\mathrm{j}} \widetilde{\mathrm{B}}_{\mathrm{j}}^{\prime \prime \prime}\left(\mathrm{x}_{\mathrm{i}}\right)\right\}
\end{array}\right] \\
& +\frac{a_{1}\left(x_{i}\right)}{h^{4}}\left[\begin{array}{c}
\left\{\mathrm{w}^{\prime \prime \prime}\left(\mathrm{x}_{\mathrm{i}+2}\right)+\sum_{\mathrm{j}=2}^{\mathrm{n}-3} \alpha_{\mathrm{j}} \widetilde{\mathrm{B}}_{\mathrm{j}}^{\prime \prime \prime}\left(\mathrm{x}_{\mathrm{i}+2}\right)\right\}-4\left\{\mathrm{w}^{\prime \prime \prime}\left(\mathrm{x}_{\mathrm{i}+1}\right)+\sum_{\mathrm{j}=2}^{\mathrm{n}-3} \alpha_{\mathrm{j}} \widetilde{\mathrm{B}}_{\mathrm{j}}^{\prime \prime \prime}\left(\mathrm{x}_{\mathrm{i}+1}\right)\right\} \\
+6\left\{\mathrm{w}^{\prime \prime \prime}\left(\mathrm{x}_{\mathrm{i}}\right)+\sum_{\mathrm{j}=2}^{\mathrm{n}-3} \alpha_{\mathrm{j}} \tilde{\mathrm{B}}_{\mathrm{j}}^{\prime \prime \prime}\left(\mathrm{x}_{\mathrm{i}}\right)\right\}-4\left\{\mathrm{w}^{\prime \prime \prime}\left(\mathrm{x}_{\mathrm{i}-1}\right)+\sum_{\mathrm{j}=2}^{\mathrm{n}-3} \alpha_{\mathrm{j}} \widetilde{\mathrm{B}}_{\mathrm{j}}^{\prime \prime \prime}\left(\mathrm{x}_{\mathrm{i}-1}\right)\right\} \\
+\left\{\mathrm{w}^{\prime \prime \prime}\left(\mathrm{x}_{\mathrm{i}-2}\right)+\sum_{\mathrm{j}=2}^{\mathrm{n}-3} \alpha_{\mathrm{j}} \widetilde{\mathrm{B}}_{\mathrm{j}}^{\prime \prime \prime}\left(\mathrm{x}_{\mathrm{i}-2}\right)\right\}
\end{array}\right] \\
& +\frac{a_{2}\left(x_{i}\right)}{2 h^{3}}\left[\begin{array}{c}
\left\{\mathrm{w}^{\prime \prime \prime}\left(\mathrm{x}_{\mathrm{i}+2}\right)+\sum_{\mathrm{j}=2}^{\mathrm{n}-3} \alpha_{\mathrm{j}} \widetilde{\mathrm{B}}_{\mathrm{j}}^{\prime \prime \prime}\left(\mathrm{x}_{\mathrm{i}+2}\right)\right\}-2\left\{\mathrm{w}^{\prime \prime \prime}\left(\mathrm{x}_{\mathrm{i}+1}\right)+\sum_{\mathrm{j}=2}^{\mathrm{n}-3} \alpha_{\mathrm{j}} \tilde{\mathrm{B}}_{\mathrm{j}}^{\prime \prime \prime}\left(\mathrm{x}_{\mathrm{i}+1}\right)\right\} \\
+2\left\{\mathrm{w}^{\prime \prime \prime}\left(\mathrm{x}_{\mathrm{i}-1}\right)+\sum_{\mathrm{j}=2}^{\mathrm{n}-3} \alpha_{\mathrm{j}} \tilde{\mathrm{B}}_{\mathrm{j}}^{\prime \prime \prime}\left(\mathrm{x}_{\mathrm{i}-1}\right)\right\}-\left\{\mathrm{w}^{\prime \prime \prime}\left(\mathrm{x}_{\mathrm{i}-2}\right)+\sum_{\mathrm{j}=2}^{\mathrm{n}-3} \alpha_{\mathrm{j}} \tilde{\mathrm{B}}_{\mathrm{j}}{ }^{\prime \prime \prime}\left(\mathrm{x}_{\mathrm{i}-2}\right)\right\}
\end{array}\right] \\
& +\frac{a_{3}\left(x_{i}\right)}{h^{2}}\left[\begin{array}{c}
\left\{\mathrm{w}^{\prime \prime \prime}\left(\mathrm{x}_{\mathrm{i}+1}\right)+\sum_{\mathrm{j}=2}^{\mathrm{n}-3} \alpha_{\mathrm{j}} \widetilde{\mathrm{B}}_{\mathrm{j}}^{\prime \prime \prime}\left(\mathrm{x}_{\mathrm{i}+1}\right)\right\}-2\left\{\mathrm{w}^{\prime \prime \prime}\left(\mathrm{x}_{\mathrm{i}}\right)+\sum_{\mathrm{j}=2}^{\mathrm{n}-3} \alpha_{\mathrm{j}} \widetilde{\mathrm{B}}_{\mathrm{j}}^{\prime \prime \prime}\left(\mathrm{x}_{\mathrm{i}}\right)\right\} \\
+\left\{\mathrm{w}^{\prime \prime \prime}\left(\mathrm{x}_{\mathrm{i}-1}\right)+\sum_{\mathrm{j}=2}^{\mathrm{n}-3} \alpha_{\mathrm{j}} \widetilde{\mathrm{B}}_{\mathrm{j}}^{\prime \prime \prime}\left(\mathrm{x}_{\mathrm{i}-1}\right)\right\}
\end{array}\right] \\
& +\frac{a_{4}\left(x_{i}\right)}{2 h}\left[\left\{w^{\prime \prime \prime}\left(\mathrm{x}_{\mathrm{i}+1}\right)+\sum_{\mathrm{j}=2}^{\mathrm{n}-3} \alpha_{\mathrm{j}} \tilde{\mathrm{B}}_{\mathrm{j}}^{\prime \prime \prime}\left(\mathrm{x}_{\mathrm{i}+1}\right)\right\}-\left\{\mathrm{w}^{\prime \prime \prime}\left(\mathrm{x}_{\mathrm{i}-1}\right)+\sum_{\mathrm{j}=2}^{\mathrm{n}-3} \alpha_{\mathrm{j}} \tilde{\mathrm{B}}_{\mathrm{j}}^{\prime \prime \prime}\left(\mathrm{x}_{\mathrm{i}-1}\right)\right\}\right] \\
& +\mathrm{a}_{5}\left(\mathrm{x}_{\mathrm{i}}\right)\left[\mathrm{w}^{\prime \prime \prime}\left(\mathrm{x}_{\mathrm{i}}\right)+\sum_{\mathrm{j}=2}^{\mathrm{n}-3} \alpha_{\mathrm{j}} \widetilde{\mathrm{B}}_{\mathrm{j}}^{\prime \prime \prime}\left(\mathrm{x}_{\mathrm{i}}\right)\right]+a_{6}\left(x_{i}\right)\left[\mathrm{w}^{\prime \prime}\left(\mathrm{x}_{\mathrm{i}}\right)+\sum_{\mathrm{j}=2}^{\mathrm{n}-3} \alpha_{\mathrm{j}} \tilde{\mathrm{B}}_{\mathrm{j}}^{\prime \prime}\left(\mathrm{x}_{\mathrm{i}}\right)\right] \\
& +a_{7}\left(x_{i}\right)\left[\mathrm{w}^{\prime}\left(\mathrm{x}_{\mathrm{i}}\right)+\sum_{\mathrm{j}=2}^{\mathrm{n}-3} \alpha_{\mathrm{j}} \tilde{\mathrm{B}}_{\mathrm{j}}^{\prime}\left(\mathrm{x}_{\mathrm{i}}\right)\right]+a_{8}\left(x_{i}\right)\left[w\left(x_{i}\right)+\sum_{j=2}^{n-2} \alpha_{j} \tilde{B}_{j}\left(x_{i}\right)\right] \\
& =b\left(x_{i}\right) \quad \text { for } \mathrm{i}=3,4, \ldots, \mathrm{n}-3 \text {. }
\end{aligned}
$$

Rearranging the terms and writing the system of equations (24) and (25) in the matrix form, we get $\mathrm{A} \alpha=\mathrm{B}$

where

$$
\begin{aligned}
& \mathrm{A}=\left[\mathrm{a}_{\mathrm{ij}}\right] \\
& \mathrm{a}_{\mathrm{ij}}=\widetilde{\mathrm{B}}_{\mathrm{j}}^{\prime \prime \prime}\left(\mathrm{x}_{\mathrm{i}-2}\right)\left(\frac{\mathrm{a}_{1}\left(\mathrm{x}_{\mathrm{i}}\right)}{\mathrm{h}^{4}}-\frac{\mathrm{a}_{2}\left(\mathrm{x}_{\mathrm{i}}\right)}{2 \mathrm{~h}^{3}}\right) \\
& +\widetilde{\mathrm{B}}_{\mathrm{j}}^{\prime \prime \prime}\left(\mathrm{x}_{\mathrm{i}-1}\right)\left(-4 \frac{\mathrm{a}_{1}\left(\mathrm{x}_{\mathrm{i}}\right)}{\mathrm{h}^{4}}+2 \frac{\mathrm{a}_{2}\left(\mathrm{x}_{\mathrm{i}}\right)}{2 \mathrm{~h}^{3}}+\frac{\mathrm{a}_{3}\left(\mathrm{x}_{\mathrm{i}}\right)}{\mathrm{h}^{2}}-\frac{\mathrm{a}_{4}\left(\mathrm{x}_{\mathrm{i}}\right)}{2 \mathrm{~h}}\right) \\
& +\widetilde{\mathrm{B}}_{\mathrm{j}}^{\prime \prime \prime}\left(\mathrm{x}_{\mathrm{i}}\right)\left(-\frac{\mathrm{a}_{0}\left(\mathrm{x}_{\mathrm{i}}\right)}{\mathrm{h}^{5}}+6 \frac{\mathrm{a}_{1}\left(\mathrm{x}_{\mathrm{i}}\right)}{\mathrm{h}^{4}}-2 \frac{\mathrm{a}_{3}\left(\mathrm{x}_{\mathrm{i}}\right)}{\mathrm{h}^{2}}\right) \\
& +\widetilde{\mathrm{B}}_{\mathrm{j}}^{\prime \prime \prime}\left(\mathrm{x}_{\mathrm{i}+1}\right)\left(5 \frac{\mathrm{a}_{0}\left(\mathrm{x}_{\mathrm{i}}\right)}{\mathrm{h}^{5}}-4 \frac{\mathrm{a}_{1}\left(\mathrm{x}_{\mathrm{i}}\right)}{\mathrm{h}^{4}}-2 \frac{\mathrm{a}_{2}\left(\mathrm{x}_{\mathrm{i}}\right)}{2 \mathrm{~h}^{3}}+\frac{\mathrm{a}_{3}\left(\mathrm{x}_{\mathrm{i}}\right)}{\mathrm{h}^{2}}+\frac{\mathrm{a}_{4}\left(\mathrm{x}_{\mathrm{i}}\right)}{2 \mathrm{~h}}\right) \\
& +\widetilde{\mathrm{B}}_{\mathrm{j}}^{\prime \prime \prime}\left(\mathrm{x}_{\mathrm{i}+2}\right)\left(-10 \frac{\mathrm{a}_{0}\left(\mathrm{x}_{\mathrm{i}}\right)}{\mathrm{h}^{5}}+\frac{\mathrm{a}_{1}\left(\mathrm{x}_{\mathrm{i}}\right)}{\mathrm{h}^{4}}+\frac{\mathrm{a}_{2}\left(\mathrm{x}_{\mathrm{i}}\right)}{2 \mathrm{~h}^{3}}\right)+\widetilde{\mathrm{B}}_{\mathrm{j}}^{\prime \prime \prime}\left(\mathrm{x}_{\mathrm{i}+3}\right)\left(10 \frac{\mathrm{a}_{0}\left(\mathrm{x}_{\mathrm{i}}\right)}{\mathrm{h}^{5}}\right) \\
& +\widetilde{\mathrm{B}}_{\mathrm{j}}^{\prime \prime \prime}\left(\mathrm{x}_{\mathrm{i}+4}\right)\left(-5 \frac{\mathrm{a}_{0}\left(\mathrm{x}_{\mathrm{i}}\right)}{\mathrm{h}^{5}}\right)+\widetilde{\mathrm{B}}_{\mathrm{j}}^{\prime \prime \prime}\left(\mathrm{x}_{\mathrm{i}+5}\right)\left(\frac{\mathrm{a}_{0}\left(\mathrm{x}_{\mathrm{i}}\right)}{\mathrm{h}^{5}}\right)+\widetilde{\mathrm{B}}_{\mathrm{j}}^{\prime \prime \prime}\left(\mathrm{x}_{\mathrm{i}}\right) \mathrm{a}_{5}\left(\mathrm{x}_{\mathrm{i}}\right) \\
& +\widetilde{\mathrm{B}}_{\mathrm{j}}^{\prime \prime}\left(\mathrm{x}_{\mathrm{i}}\right) \mathrm{a}_{6}\left(\mathrm{x}_{\mathrm{i}}\right)+\widetilde{\mathrm{B}}_{\mathrm{j}}^{\prime}\left(\mathrm{x}_{\mathrm{i}}\right) \mathrm{a}_{7}\left(\mathrm{x}_{\mathrm{i}}\right)+\widetilde{\mathrm{B}}_{\mathrm{j}}\left(\mathrm{x}_{\mathrm{i}}\right) \mathrm{a}_{8}\left(\mathrm{x}_{\mathrm{i}}\right) \\
& \text { for } \mathrm{i}=2, \quad \mathrm{j}=2,3, \ldots, \mathrm{n}-3 . \\
& \mathrm{a}_{\mathrm{ij}}=\widetilde{\mathrm{B}}_{\mathrm{j}}^{\prime \prime}{ }^{\prime}\left(\mathrm{x}_{\mathrm{i}-3}\right)\left(-\frac{\mathrm{a}_{0}\left(\mathrm{x}_{\mathrm{i}}\right)}{2 \mathrm{~h}^{5}}\right)
\end{aligned}
$$




$$
\begin{aligned}
& +\widetilde{B}_{j}^{\prime \prime \prime}\left(x_{i-2}\right)\left(4 \frac{a_{0}\left(x_{i}\right)}{2 h^{5}}+\frac{a_{1}\left(x_{i}\right)}{h^{4}}-\frac{a_{2}\left(x_{i}\right)}{2 h^{3}}\right) \\
& +\widetilde{B}_{j}^{\prime \prime} \prime\left(x_{i-1}\right)\left(-5 \frac{a_{0}\left(x_{i}\right)}{2 h^{5}}-4 \frac{a_{1}\left(x_{i}\right)}{h^{4}}+2 \frac{a_{2}\left(x_{i}\right)}{2 h^{3}}+\frac{a_{3}\left(x_{i}\right)}{h^{2}}-\frac{a_{4}\left(x_{i}\right)}{2 h}\right) \\
& +\widetilde{B}_{j}^{\prime \prime \prime}\left(x_{i}\right)\left(6 \frac{a_{1}\left(x_{i}\right)}{h^{4}}-2 \frac{a_{3}\left(x_{i}\right)}{h^{2}}\right)+\widetilde{B}_{j}^{\prime \prime} '\left(x_{i+1}\right)\left(5 \frac{a_{0}\left(x_{i}\right)}{2 h^{5}}-4 \frac{a_{1}\left(x_{i}\right)}{h^{4}}-2 \frac{a_{2}\left(x_{i}\right)}{2 h^{3}}+\frac{a_{3}\left(x_{i}\right)}{h^{2}}+\frac{a_{4}\left(x_{i}\right)}{2 h}\right) \\
& +\widetilde{B}_{j}^{\prime \prime \prime}\left(x_{i}+2\right)\left(-4 \frac{a_{0}\left(x_{i}\right)}{2 h^{5}}+\frac{a_{1}\left(x_{i}\right)}{h^{4}}+\frac{a_{2}\left(x_{i}\right)}{2 h^{3}}\right)+\widetilde{B}_{j}^{\prime \prime \prime}\left(x_{i}+3\right)\left(\frac{a_{0}\left(x_{i}\right)}{2 h^{5}}\right) \\
& +\widetilde{B}_{j}^{\prime \prime \prime}\left(x_{i}\right) a_{5}\left(x_{i}\right)+\widetilde{B}_{j}^{\prime \prime}\left(x_{i}\right) a_{6}\left(x_{i}\right)+\widetilde{B}_{j}^{\prime}\left(x_{i}\right) a_{7}\left(x_{i}\right)+\widetilde{B}_{j}\left(x_{i}\right) a_{8}\left(x_{i}\right) \\
& \text { for } i=3,4, \ldots, n-3, \quad j=2,3, \ldots, n-3 . \\
& B=\left[b_{i}\right] ;
\end{aligned}
$$

$\mathrm{b}_{\mathrm{i}}=$

$\mathrm{b}\left(\mathrm{x}_{\mathrm{i}}\right)-$

$$
\left[\begin{array}{c}
\mathrm{w}^{\prime \prime \prime}\left(\mathrm{x}_{\mathrm{i}-2}\right)\left(\frac{\mathrm{a}_{1}\left(\mathrm{x}_{\mathrm{i}}\right)}{\mathrm{h}^{4}}-\frac{\mathrm{a}_{2}\left(\mathrm{x}_{\mathrm{i}}\right)}{2 \mathrm{~h}^{3}}\right) \\
+\mathrm{w}^{\prime \prime \prime}\left(\mathrm{x}_{\mathrm{i}-1}\right)\left(-4 \frac{\mathrm{a}_{1}\left(\mathrm{x}_{\mathrm{i}}\right)}{\mathrm{h}^{4}}+2 \frac{\mathrm{a}_{2}\left(\mathrm{x}_{\mathrm{i}}\right)}{2 \mathrm{~h}^{3}}+\frac{\mathrm{a}_{3}\left(\mathrm{x}_{\mathrm{i}}\right)}{\mathrm{h}^{2}}-\frac{\mathrm{a}_{4}\left(\mathrm{x}_{\mathrm{i}}\right)}{2 \mathrm{~h}}\right) \\
+\mathrm{w}^{\prime \prime \prime}\left(\mathrm{x}_{\mathrm{i}}\right)\left(-\frac{\mathrm{a}_{0}\left(\mathrm{x}_{\mathrm{i}}\right)}{\mathrm{h}^{5}}+6 \frac{\mathrm{a}_{1}\left(\mathrm{x}_{\mathrm{i}}\right)}{\mathrm{h}^{4}}-2 \frac{\mathrm{a}_{3}\left(\mathrm{x}_{\mathrm{i}}\right)}{\mathrm{h}^{2}}\right) \\
+\mathrm{w}^{\prime \prime \prime}\left(\mathrm{x}_{\mathrm{i}+1}\right)\left(5 \frac{\mathrm{a}_{0}\left(\mathrm{x}_{\mathrm{i}}\right)}{\mathrm{h}^{5}}-4 \frac{\mathrm{a}_{1}\left(\mathrm{x}_{\mathrm{i}}\right)}{\mathrm{h}^{4}}-2 \frac{\mathrm{a}_{2}\left(\mathrm{x}_{\mathrm{i}}\right)}{2 \mathrm{~h}^{3}}+\frac{\mathrm{a}_{3}\left(\mathrm{x}_{\mathrm{i}}\right)}{\mathrm{h}^{2}}+\frac{\mathrm{a}_{4}\left(\mathrm{x}_{\mathrm{i}}\right)}{2 \mathrm{~h}}\right) \\
+\mathrm{w}^{\prime \prime \prime}\left(\mathrm{x}_{\mathrm{i}+2}\right)\left(-10 \frac{\mathrm{a}_{0}\left(\mathrm{x}_{\mathrm{i}}\right)}{\mathrm{h}^{5}}+\frac{\mathrm{a}_{1}\left(\mathrm{x}_{\mathrm{i}}\right)}{\mathrm{h}^{4}}+\frac{\mathrm{a}_{3}\left(\mathrm{x}_{\mathrm{i}}\right)}{2 \mathrm{~h}^{3}}\right)+\mathrm{w}^{\prime \prime \prime}\left(\mathrm{x}_{\mathrm{i}+3}\right)\left(10 \frac{\mathrm{a}_{0}\left(\mathrm{x}_{\mathrm{i}}\right)}{\mathrm{h}^{5}}\right)+\mathrm{w}^{\prime \prime \prime}\left(\mathrm{x}_{\mathrm{i}+4}\right)\left(-5 \frac{\mathrm{a}_{0}\left(\mathrm{x}_{\mathrm{i}}\right)}{\mathrm{h}^{5}}\right) \\
+\mathrm{w}^{\prime \prime \prime}\left(\mathrm{x}_{\mathrm{i}+5}\right)\left(\frac{\mathrm{a}_{0}\left(\mathrm{x}_{\mathrm{i}}\right)}{\mathrm{h}^{5}}\right)+\mathrm{w}^{\prime \prime \prime}\left(\mathrm{x}_{\mathrm{i}}\right) \mathrm{a}_{5}\left(\mathrm{x}_{\mathrm{i}}\right)+\mathrm{w}^{\prime \prime}\left(\mathrm{x}_{\mathrm{i}}\right) \mathrm{a}_{6}\left(\mathrm{x}_{\mathrm{i}}\right)+\mathrm{w}^{\prime}\left(\mathrm{x}_{\mathrm{i}}\right) \mathrm{a}_{7}\left(\mathrm{x}_{\mathrm{i}}\right) \\
+\mathrm{w}\left(\mathrm{x}_{\mathrm{i}}\right) \mathrm{a}_{8}\left(\mathrm{x}_{\mathrm{i}}\right)
\end{array}\right.
$$

for $i=2$.

$b_{\mathrm{i}}$

$$
=\mathrm{b}\left(\mathrm{x}_{\mathrm{i}}\right)
$$

$$
-\left[\begin{array}{c}
w^{\prime \prime \prime}\left(\mathrm{x}_{\mathrm{i}-3}\right)\left(-\frac{\mathrm{a}_{0}\left(\mathrm{x}_{\mathrm{i}}\right)}{2 \mathrm{~h}^{5}}\right) \\
+\mathrm{w}^{\prime \prime \prime}\left(\mathrm{x}_{\mathrm{i}-2}\right)\left(4 \frac{\mathrm{a}_{0}\left(\mathrm{x}_{\mathrm{i}}\right)}{2 \mathrm{~h}^{5}}+\frac{\mathrm{a}_{1}\left(\mathrm{x}_{\mathrm{i}}\right)}{\mathrm{h}^{4}}-\frac{\mathrm{a}_{2}\left(\mathrm{x}_{\mathrm{i}}\right)}{2 \mathrm{~h}^{3}}\right) \\
+\mathrm{w}^{\prime \prime \prime}\left(\mathrm{x}_{\mathrm{i}-1}\right)\left(-5 \frac{\mathrm{a}_{0}\left(\mathrm{x}_{\mathrm{i}}\right)}{2 \mathrm{~h}^{5}}-4 \frac{\mathrm{a}_{1}\left(\mathrm{x}_{\mathrm{i}}\right)}{\mathrm{h}^{4}}+2 \frac{\mathrm{a}_{2}\left(\mathrm{x}_{\mathrm{i}}\right)}{2 \mathrm{~h}^{3}}+\frac{\mathrm{a}_{3}\left(\mathrm{x}_{\mathrm{i}}\right)}{\mathrm{h}^{2}}-\frac{\mathrm{a}_{4}\left(\mathrm{x}_{\mathrm{i}}\right)}{2 \mathrm{~h}}\right) \\
+\mathrm{w}^{\prime \prime \prime}\left(\mathrm{x}_{\mathrm{i}}\right)\left(6 \frac{\mathrm{a}_{1}\left(\mathrm{x}_{\mathrm{i}}\right)}{\mathrm{h}^{4}}-2 \frac{\mathrm{a}_{3}\left(\mathrm{x}_{\mathrm{i}}\right)}{\mathrm{h}^{2}}\right)+\mathrm{w}^{\prime \prime \prime}\left(\mathrm{x}_{\mathrm{i}+1}\right)\left(5 \frac{\mathrm{a}_{0}\left(\mathrm{x}_{\mathrm{i}}\right)}{2 \mathrm{~h}^{5}}-4 \frac{\mathrm{a}_{1}\left(\mathrm{x}_{\mathrm{i}}\right)}{\mathrm{h}^{4}}-2 \frac{\mathrm{a}_{2}\left(\mathrm{x}_{\mathrm{i}}\right)}{2 \mathrm{~h}^{3}}+\frac{\mathrm{a}_{3}\left(\mathrm{x}_{\mathrm{i}}\right)}{\mathrm{h}^{2}}+\frac{\mathrm{a}_{4}\left(\mathrm{x}_{\mathrm{i}}\right)}{2 \mathrm{~h}}\right) \\
+\mathrm{w}^{\prime \prime \prime}\left(\mathrm{x}_{\mathrm{i}+2}\right)\left(-4 \frac{\mathrm{a}_{0}\left(\mathrm{x}_{\mathrm{i}}\right)}{2 \mathrm{~h}^{5}}+\frac{\mathrm{a}_{1}\left(\mathrm{x}_{\mathrm{i}}\right)}{\mathrm{h}^{4}}+\frac{\mathrm{a}_{2}\left(\mathrm{x}_{\mathrm{i}}\right)}{2 \mathrm{~h}^{3}}\right)+\mathrm{w}^{\prime \prime \prime}\left(\mathrm{x}_{\mathrm{i}+3}\right)\left(\frac{\mathrm{a}_{0}\left(\mathrm{x}_{\mathrm{i}}\right)}{\left.2 \mathrm{~h}^{5}\right)}\right) \\
+\mathrm{w}^{\prime \prime \prime}\left(\mathrm{x}_{\mathrm{i}}\right) \mathrm{a}_{5}\left(\mathrm{x}_{\mathrm{i}}\right)+\mathrm{w}^{\prime \prime}\left(\mathrm{x}_{\mathrm{i}}\right) \mathrm{a}_{6}\left(\mathrm{x}_{\mathrm{i}}\right)+\mathrm{w}^{\prime}\left(\mathrm{x}_{\mathrm{i}}\right) \mathrm{a}_{7}\left(\mathrm{x}_{\mathrm{i}}\right)+\mathrm{w}\left(\mathrm{x}_{\mathrm{i}}\right) \mathrm{a}_{8}\left(\mathrm{x}_{\mathrm{i}}\right) \\
\text { for } \mathrm{i}=3,4, \ldots, \mathrm{n}-3
\end{array}\right.
$$

and

$$
\alpha=\left[\alpha_{2}, \alpha_{3}, \ldots, \alpha_{n-3}\right]^{T} .
$$

\section{Solution Procedure to Find the Nodal Parameters}

The basis function $\widetilde{\mathrm{B}_{\mathrm{i}}}(\mathrm{x})$ is defined only in the interval $\left[\mathrm{x}_{\mathrm{i}-2}, \mathrm{x}_{\mathrm{i}+3}\right]$ and outside of this interval it is zero. Also at the end points of the interval $\left[\mathrm{x}_{\mathrm{i}-2}, \mathrm{x}_{\mathrm{i}+3}\right]$ the basis function $\widetilde{\mathrm{B}_{\mathrm{i}}}(\mathrm{x})$ vanishes. Therefore, 
$\widetilde{\mathrm{B}_{i}}(\mathrm{x})$ is having non-vanishing values at the mesh points $\mathrm{x}_{\mathrm{i}-1}, \mathrm{x}_{\mathrm{i}}, \mathrm{x}_{\mathrm{i}+1}, \mathrm{x}_{\mathrm{i}+2}$ and zero at the other mesh points. The first three derivatives of $\widetilde{\mathrm{B}_{\mathrm{i}}}(\mathrm{x})$ also have the same nature at the mesh points as in the case of $\widetilde{\mathrm{B}_{\mathrm{i}}}(\mathrm{x})$. Using these facts, we can say that the matrix A defined in (27) is a 12-band matrix. Therefore, the system of equations (26) is a 12-band system in $\alpha_{i}^{\prime}$ s. The nodal parameters $\alpha_{i}^{\prime} \mathrm{s}$ can be obtained by using band matrix solution package. We have used the FORTRAN-90 programming to solve the boundary value problem (1) - (2) by the proposed method.

\section{NUMERICAL EXAMPLES}

To demonstrate the applicability of the proposed method for solving the eighth order boundary value problems of type (1) - (2), we considered five examples of which three are linear and two are non linear boundary value problems. Numerical results for each problem are presented in tabular forms and compared with the exact solutions available in the literature.

Example 1 Consider the linear boundary value problem

$\mathrm{y}^{(8)}-\mathrm{y}=-8 \mathrm{e}^{\mathrm{x}}, \quad 0<x<1$

subject to the boundary conditions

$y(0)=1, y(1)=0, y^{\prime}(0)=0, y^{\prime}(1)=-e$,

$\mathrm{y}^{\prime \prime}(0)=-1, \mathrm{y}^{\prime \prime}(1)=-2 \mathrm{e}, \mathrm{y}^{\prime \prime \prime}(0)=-2, \mathrm{y}^{\prime \prime \prime}(1)=-3 \mathrm{e}$.

The exact solution for the above problem is given by $y(x)=(1-x) e^{x}$. The proposed method is tested on this problem where the domain $[0,1]$ is divided into 12 equal subintervals. Numerical results for this problem are shown in Table 1 . The maximum absolute error obtained by the proposed method is $2.336502 \times 10^{-5}$.

Table 1. Numerical results for Example 1

\begin{tabular}{|l|c|c|}
\hline$x$ & Exact Solution & Absolute error by proposed method \\
\hline 0.1 & $9.946538 \mathrm{E}-01$ & $1.430511 \mathrm{E}-06$ \\
\hline 0.2 & $9.771222 \mathrm{E}-01$ & $2.503395 \mathrm{E}-06$ \\
\hline 0.3 & $9.449012 \mathrm{E}-01$ & $1.019239 \mathrm{E}-05$ \\
\hline 0.4 & $8.950948 \mathrm{E}-01$ & $1.895428 \mathrm{E}-05$ \\
\hline 0.5 & $8.243606 \mathrm{E}-01$ & $2.336502 \mathrm{E}-05$ \\
\hline 0.6 & $7.288475 \mathrm{E}-01$ & $2.181530 \mathrm{E}-05$ \\
\hline 0.7 & $6.041259 \mathrm{E}-01$ & $1.412630 \mathrm{E}-05$ \\
\hline 0.8 & $4.451082 \mathrm{E}-01$ & $6.496906 \mathrm{E}-06$ \\
\hline 0.9 & $2.459602 \mathrm{E}-01$ & $1.132488 \mathrm{E}-06$ \\
\hline
\end{tabular}

Example 2 Consider the linear boundary value problem

$\mathrm{y}^{(8)}+\mathrm{xy}=-\left(48+15 \mathrm{x}+\mathrm{x}^{3}\right) \mathrm{e}^{\mathrm{x}}, \quad 0<x<1$

subject to the boundary conditions

$\mathrm{y}(0)=0, \mathrm{y}(1)=0, \mathrm{y}^{\prime}(0)=1, \mathrm{y}^{\prime}(1)=-\mathrm{e}$,

$\mathrm{y}^{\prime \prime}(0)=0, \mathrm{y}^{\prime \prime}(1)=-4 \mathrm{e}, \quad \mathrm{y}^{\prime \prime \prime}(0)=-3, \mathrm{y}^{\prime \prime \prime}(1)=-9 \mathrm{e}$.

The exact solution for the above problem is given by $\mathrm{y}(\mathrm{x})=\mathrm{x}(1-\mathrm{x}) \mathrm{e}^{\mathrm{x}}$. The proposed method is tested on this problem where the domain $[0,1]$ is divided into 12 equal subintervals. Numerical results for this problem are shown in Table 2. The maximum absolute error obtained by the proposed method is $5.340576 \times 10^{-5}$.

Table 2. Numerical results for Example 2

\begin{tabular}{|l|l|r|}
\hline$x$ & Exact Solution & Absolute error by proposed method \\
\hline 0.1 & $9.946539 \mathrm{E}-02$ & $9.313226 \mathrm{E}-07$ \\
\hline 0.2 & $1.954244 \mathrm{E}-01$ & $9.834766 \mathrm{E}-07$ \\
\hline
\end{tabular}


Quartic B-Spline Collocation Method for Eighth Order Boundary Value Problems

\begin{tabular}{|l|l|r|}
\hline 0.3 & $2.834704 \mathrm{E}-01$ & $1.129508 \mathrm{E}-05$ \\
\hline 0.4 & $3.580379 \mathrm{E}-01$ & $2.971292 \mathrm{E}-05$ \\
\hline 0.5 & $4.121803 \mathrm{E}-01$ & $4.711747 \mathrm{E}-05$ \\
\hline 0.6 & $4.373085 \mathrm{E}-01$ & $5.340576 \mathrm{E}-05$ \\
\hline 0.7 & $4.228881 \mathrm{E}-01$ & $4.258752 \mathrm{E}-05$ \\
\hline 0.8 & $3.560865 \mathrm{E}-01$ & $2.211332 \mathrm{E}-05$ \\
\hline 0.9 & $2.213642 \mathrm{E}-01$ & $4.261732 \mathrm{E}-06$ \\
\hline
\end{tabular}

Example 3 Consider the linear boundary value problem

$y^{(8)}+y^{(7)}+2 y^{(6)}+2 y^{(5)}+2 y^{(4)}+2 y^{\prime \prime \prime}+2 y^{\prime \prime}+y^{\prime}+y=14 \cos x-4 x \sin x-16 \sin x, \quad 0<x<1$

subject to the boundary conditions

$\mathrm{y}(0)=0, \mathrm{y}(1)=0, \mathrm{y}^{\prime}(0)=-1, \mathrm{y}^{\prime}(1)=2 \sin 1$,

$\mathrm{y}^{\prime \prime}(0)=0, \mathrm{y}^{\prime \prime}(1)=4 \cos 1+2 \sin 1, \quad \mathrm{y}^{\prime \prime \prime}(0)=7, \quad \mathrm{y}^{\prime \prime \prime}(1)=6 \cos 1-6 \sin 1$.

The exact solution for the above problem is given by $y(x)=\left(x^{2}-1\right) \sin x$. The proposed method is tested on this problem where the domain $[0,1]$ is divided into 12 equal subintervals. Numerical results for this problem are shown in Table 3. The maximum absolute error obtained by the proposed method is $2.852082 \times 10^{-5}$.

Table 3. Numerical results for Example 3

\begin{tabular}{|l|l|r|}
\hline$x$ & Exact Solution & Absolute error by proposed method \\
\hline 0.1 & $-9.883508 \mathrm{E}-02$ & $1.050532 \mathrm{E}-06$ \\
\hline 0.2 & $-1.907226 \mathrm{E}-01$ & $8.866191 \mathrm{E}-06$ \\
\hline 0.3 & $-2.689234 \mathrm{E}-01$ & $2.023578 \mathrm{E}-05$ \\
\hline 0.4 & $-3.271114 \mathrm{E}-01$ & $2.852082 \mathrm{E}-05$ \\
\hline 0.5 & $-3.595692 \mathrm{E}-01$ & $2.828240 \mathrm{E}-05$ \\
\hline 0.6 & $-3.613712 \mathrm{E}-01$ & $2.050400 \mathrm{E}-05$ \\
\hline 0.7 & $-3.285510 \mathrm{E}-01$ & $9.685755 \mathrm{E}-06$ \\
\hline 0.8 & $-2.582482 \mathrm{E}-01$ & $2.682209 \mathrm{E}-06$ \\
\hline 0.9 & $-1.488321 \mathrm{E}-01$ & $1.639128 \mathrm{E}-07$ \\
\hline
\end{tabular}

Example 4 Consider the nonlinear boundary value problem

$\mathrm{y}^{(8)}=\mathrm{e}^{-\mathrm{x}} \mathrm{y}^{2}(\mathrm{x}), \quad 0<x<1$

subject to the boundary conditions

$\mathrm{y}(0)=0, \mathrm{y}(1)=\mathrm{e}, \mathrm{y}^{\prime}(0)=1, \mathrm{y}^{\prime}(1)=\mathrm{e}$,

$y^{\prime \prime}(0)=1, y^{\prime \prime}(1)=e, \quad y^{\prime \prime \prime}(0)=1, y^{\prime \prime \prime}(1)=e$.

The exact solution for the above problem is given by $y(x)=e^{x}$. This nonlinear boundary value problem is converted into a sequence of linear boundary value problems generated by quasi linearization technique [23] as

$\mathrm{y}_{(\mathrm{n}+1)}^{(8)}+\left[-2 \mathrm{e}^{-\mathrm{x}} \mathrm{y}_{(\mathrm{n})}\right] \mathrm{y}_{(\mathrm{n}+1)}=-\mathrm{e}^{-\mathrm{x}} \mathrm{y}_{(\mathrm{n})}^{2} \quad$ for $\mathrm{n}=0,1,2, \ldots$

subject to the boundary conditions

$\mathrm{y}_{(\mathrm{n}+1)}(0)=1, \mathrm{y}_{(\mathrm{n}+1)}(1)=\mathrm{e}, \mathrm{y}_{(\mathrm{n}+1)}^{\prime}(0)=1, \mathrm{y}_{(\mathrm{n}+1)}^{\prime}(1)=\mathrm{e}$,

$y_{(n+1)}^{\prime \prime}(0)=1, \quad y_{(n+1)}^{\prime \prime}(1)=e, \quad y_{(n+1)}^{\prime \prime \prime}(0)=1, y_{(n+1)}^{\prime \prime \prime}(1)=e$.

Here $y_{(n+1)}$ is the $(n+1)^{\text {th }}$ approximation for $y$. The domain $[0,1]$ is divided into 12 equal subintervals and the proposed method is applied to the sequence of problems [37]. Numerical results 
Quartic B-Spline Collocation Method for Eighth Order Boundary Value Problems

for this problem are presented in Table 4. The maximum absolute error obtained by the proposed method is $3.123283 \times 10^{-5}$.

Table 4. Numerical results for Example 4

\begin{tabular}{|l|c|r|}
\hline$x$ & Exact Solution & Absolute error by proposed method \\
\hline 0.1 & 1.105171 & $2.384186 \mathrm{E}-06$ \\
\hline 0.2 & 1.221403 & $5.960464 \mathrm{E}-07$ \\
\hline 0.3 & 1.349859 & $3.695488 \mathrm{E}-06$ \\
\hline 0.4 & 1.491825 & $2.741814 \mathrm{E}-06$ \\
\hline 0.5 & 1.648721 & $5.602837 \mathrm{E}-06$ \\
\hline 0.6 & 1.822119 & $1.478195 \mathrm{E}-05$ \\
\hline 0.7 & 2.013753 & $2.408028 \mathrm{E}-05$ \\
\hline 0.8 & 2.225541 & $1.382828 \mathrm{E}-05$ \\
\hline 0.9 & 2.459603 & $3.123283 \mathrm{E}-05$ \\
\hline
\end{tabular}

Example 5 Consider the nonlinear boundary value problem

$\mathrm{y}^{(8)}=7 !\left[\mathrm{e}^{-8 \mathrm{y}}-\frac{2}{(1+\mathrm{x})^{8}}\right], \quad 0<x<e^{1 / 2}-1$

subject to the boundary conditions

$\mathrm{y}(0)=0, \mathrm{y}\left(e^{1 / 2}-1\right)=1 / 2, \mathrm{y}^{\prime}(0)=1, \mathrm{y}^{\prime}\left(e^{1 / 2}-1\right)=\frac{1}{e^{1 / 2}}$,

$\mathrm{y}^{\prime \prime}(0)=-1, \mathrm{y}^{\prime \prime}\left(e^{1 / 2}-1\right)=-\frac{1}{\mathrm{e}^{\prime}}, \quad \mathrm{y}^{\prime \prime \prime}(0)=2, \mathrm{y}^{\prime \prime \prime}\left(e^{1 / 2}-1\right)=\frac{2}{\mathrm{e}^{3 / 2}}$.

The exact solution for the above problem is given by $\mathrm{y}(\mathrm{x})=\ln (1+\mathrm{x})$. This nonlinear boundary value problem is converted into a sequence of linear boundary value problems generated by quasi linearization technique [23] as

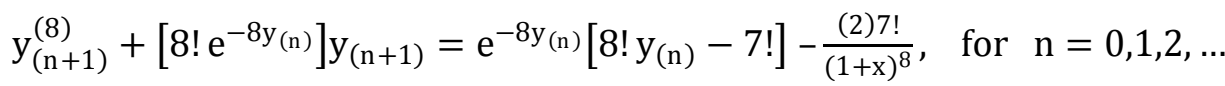

subject to the boundary conditions

$\mathrm{y}_{(\mathrm{n}+1)}(0)=0, \quad \mathrm{y}_{(\mathrm{n}+1)}\left(e^{1 / 2}-1\right)=1 / 2, \mathrm{y}_{(\mathrm{n}+1)}^{\prime}(0)=1, \mathrm{y}_{(\mathrm{n}+1)}^{\prime}\left(e^{1 / 2}-1\right)=\frac{1}{e^{1 / 2}}$,

$\mathrm{y}_{(\mathrm{n}+1)}^{\prime \prime}(0)=-1, \quad \mathrm{y}_{(\mathrm{n}+1)}^{\prime \prime}\left(e^{1 / 2}-1\right)=-\frac{1}{\mathrm{e}^{\prime}}, \quad \mathrm{y}_{(\mathrm{n}+1)}^{\prime \prime \prime}(0)=2, \quad \mathrm{y}_{(\mathrm{n}+1)}^{\prime \prime \prime}\left(e^{1 / 2}-1\right)=\frac{2}{\mathrm{e}^{3 / 2}}$.

Here $\mathrm{y}_{(\mathrm{n}+1)}$ is the $(\mathrm{n}+1)^{\mathrm{th}}$ approximation for $\mathrm{y}$. The domain $\left[0, e^{\frac{1}{2}}-1\right]$ is divided into 12 equal subintervals and the proposed method is applied to the sequence of problems [41]. Numerical results for this problem are presented in Table 5 . The maximum absolute error obtained by the proposed method is $4.261732 \times 10^{-6}$.

Table 5. Numerical results for Example 5

\begin{tabular}{|c|c|r|}
\hline$x$ & Exact Solution & Absolute error by proposed method \\
\hline $6.487213 \mathrm{E}-02$ & $6.285473 \mathrm{E}-02$ & $5.215406 \mathrm{E}-08$ \\
\hline $1.297443 \mathrm{E}-01$ & $1.219913 \mathrm{E}-01$ & $2.682209 \mathrm{E}-07$ \\
\hline $1.946164 \mathrm{E}-01$ & $1.778251 \mathrm{E}-01$ & $1.356006 \mathrm{E}-06$ \\
\hline $2.594885 \mathrm{E}-01$ & $2.307057 \mathrm{E}-01$ & $3.203750 \mathrm{E}-06$ \\
\hline $3.243607 \mathrm{E}-01$ & $2.809298 \mathrm{E}-01$ & $4.261732 \mathrm{E}-06$ \\
\hline $3.892328 \mathrm{E}-01$ & $3.287517 \mathrm{E}-01$ & $3.606081 \mathrm{E}-06$ \\
\hline $4.541049 \mathrm{E}-01$ & $3.743905 \mathrm{E}-01$ & $1.966953 \mathrm{E}-06$ \\
\hline $5.189770 \mathrm{E}-01$ & $4.180371 \mathrm{E}-01$ & $4.768372 \mathrm{E}-07$ \\
\hline $5.838492 \mathrm{E}-01$ & $4.598581 \mathrm{E}-01$ & $7.152557 \mathrm{E}-07$ \\
\hline & & \\
\hline
\end{tabular}




\section{CONCLusions}

In this paper, we have developed a collocation method with quartic B-splines as basis functions to solve eighth order boundary value problems. Here we have taken internal mesh points $\mathrm{x}_{2}, \mathrm{x}_{3}, \ldots, \mathrm{x}_{\mathrm{n}-3}$ as the selected collocation points. The quartic B-spline basis set has been redefined into a new set of basis functions which in number match with the number of selected collocation points. The proposed method is applied to solve several number of linear and non-linear problems to test the efficiency of the method. The numerical results obtained by the proposed method are in good agreement with the exact solutions available in the literature. The objective of this paper is to present a simple method to solve a eighth order boundary value problem and its easiness for implementation.

\section{REFERENCES}

[1] Davies, A. R., Karageorghis, A., and Phillips, T. N. Spectral Galerkin methods for the primary two point boundary-value problem in modelling viscoelastic flow. International Journal of Numerical Methods, 26: 647-662(1988).

[2] Karageorghis, A., Phillips, T. N., and Davies, A. R. Spectral collocation methods for the primary two point boundary-value problem in modelling viscoelastic flows. Inter-national Journal of Numerical Methods in Engineering, 26: 805-813(1988).

[3] Chandrasekhar S., Hydrodynamics and Hydromagnetic Stability, Dover, New York(1981).

[4] Bishop, R. E. D., Cannon S. M., and Miao, S. On coupled bending and torsional vibration of uniform beams. Sound Vibration, 131:457-464(1989).

[5] Agarwal, R.P., Boundary value problems for higher-order differential equations, World Scientific, Singapore(1986).

[6] Boutayeb, A., and Twizell, E. H. Finite-difference methods for the solution of eighth-order boundary-value problems. Applied Mathematics Letters, 48:63-75(1993).

[7] Twizell, E. H., Boutayeb, A., and Djidjeli, K. Numerical methods for eighth, tenth and twelfth-order eigenvalue problems arising in thermal instability. Adv. Comput. Math., 2:407-436(1994).

[8] Twizell, E. H., and Siddiqi, S. S. Spline solution of linear eighth-order boundary value problems. Comput. Methods Appl. Mech. Eng., 131:309-325(1996).

[9] Inc, M., and Evans, D. J. An efficient approach to approximate solutions of eighth-order boundary-value problems. Int. J. Comput. Math., 81:685-692(2004).

[10] Shahid, Siddiqi, S., and Ghazala Akram. Nonic spline solutions of eighth order boundary value problems. J. Comput. Appl. Math., 182:829-845(2006).

[11] Shahid, Siddiqi, S., and Ghazala Akram. Solutions of eighth order boundary value problems using nonpolynomial spline technique. International Journal of Computer Mathematics, 84(3):347-368(2007).

[12] Scott, M. R., and Watts, H. A. Computional solution of linear two points bvp via orthonormalization. SIMA J. Numer. Anel., 14:40-70(1977).

[13] Scott, M. R., and Watts, H. A. A systematized collection of codes for solving two-point bvps, numerical methods for differential systems, Academic Press, (1976).

[14] Scott Layne Watson, M. R. Solving spline-collocation approximations to nonlinear two-point boundary value problems by a homotopy method. Appl. Math. Comput., 24:333-357(1987).

[15] Liu, G. R., and Wu, T. Y. Differential quadrature solutions of eighth order boundary value differential equations. J. Comput. Appl. Math., 145:223-235(1973).

[16] He, J. H. Approximate analytical solution for seepage flow with fractional derivatives in porous media. Comput. Methods Appl. Mech. Eng., 167(1-2):57-68(1998).

[17] He, J. H. Variational iteration method a kind of nonlinear analytical technique: some examples. Int. J. Nonlinear Mech., 34:699-708(1999).

[18] He, J. H. Variational method for autonomous ordinary differential equations. Appl. Math. Comput., 114:115-123(2000).

[19] He, J. H. Variational theory for linear magneto-elasticity. Int. J. Nonlinear Sci. Numer. Simul., 2:309$316(2001)$.

[20] He, J. H. Variational principle for some nonlinear partial differential equations with variables coefficients. Chaos Solitons Fract., 19:847-851(2004).

[21] Wazwaz, A. M. Approximate solutions to boundary value problems of higher order by the modified decomposition method. Comput. Math. Appl., 40:679-691(2000).

[22] Wazwaz, A. M. The numerical solution of special eighth-order boundary value problems by the modified decomposition method. Neural, Parallel and Scientific Computations, 8(2):133-146(2000). 
[23] Bellman R.E., and Kalaba R.E., Quasi linearization and Nonlinear Boundary value problems, American Elsevier, New York (1965).

[24] Reddy J.N., An introduction to the Finite Element Method, Tata Mc-GrawHill Publishing Company Limited, 3rd Edition, New Delhi(2005).

[25] Prenter P.M., Splines and Variational Methods, John-Wiley and Sons, New York(1989).

[26] Carl de Boor, A Practical Guide to Splines, Springer-Verlag(1978) .

[27] Schoenberg I.J., On Spline Functions, University of Wisconsin, MRC Report 625(1966).

\section{AUTHORS' BIOGRAPHY}

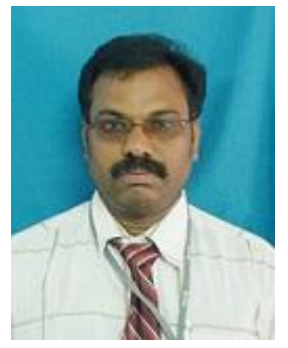

The author completed his master's degree from Pondicherry University during the academic years 1995-1997. He obtained his PhD degree from NIT-Warangal during the year 2014. His area of interest is Finite Element Methods. At present he is working at Department of Computational Sciences as Associate professor, Wollega University, Nekemte, Ethiopia. The author has University level experience to teach both UG and PG level courses.

Citation: Y. S. Raju, "Quartic B-Spline Collocation Method for Eighth Order Boundary Value Problems ", International Journal of Scientific and Innovative Mathematical Research, vol. 5, no. 8, p. 15-26, 2017., http://dx.doi.org/10.20431/2347-3142.0508003

Copyright: ( 92017 Authors. This is an open-access article distributed under the terms of the Creative Commons Attribution License, which permits unrestricted use, distribution, and reproduction in any medium, provided the original author and source are credited. 